Please quote as: Bahle, G.; Calma, A.; Leimeister, J. M.; Lukowicz, P.; Oeste-Reiß, S.; Reitmaier, T.; Schmidt, A.; Sick, B.; Stumme, G. \& Zweig, K. (2016): Lifelong Learning and Collaboration of Smart Technical Systems in Open-Ended Environments - Opportunistic Collaborative Interactive Learning. In: International Conference on Autonomic Computing, Würzburg, Germany. 


\title{
Lifelong Learning and Collaboration of Smart Technical Systems in Open-Ended Environments - Opportunistic Collaborative Interactive Learning
}

\author{
Gernot Bahle ${ }^{\ddagger}$, Adrian Calma*, Jan Marco Leimeister ${ }^{\dagger * *}$, Paul Lukowicz ${ }^{\ddagger}$, Sarah Oeste-Rei $\beta^{\dagger}$, \\ Tobias Reitmaier*, Albrecht Schmidt ${ }^{\S}$, Bernhard Sick*, Gerd Stumme ${ }^{\Uparrow}$, and Katharina Anna Zweig\| \\ $\ddagger$ Embedded Intelligence, DFKI, Kaiserslautern, Germany, Email: \{gernot.bahle|paul.lukowicz\}@ dfki.de \\ * Intelligent Embedded Systems (IES), Univ. of Kassel, Germany, Email: \{adrian.calma|tobias.reitmaier|bsick\}@uni-kassel.de \\ $\dagger$ Research Center for IS Design (ITeG), Univ. of Kassel, Germany, Email: \{leimeister|oeste-reiss\}@uni-kassel.de \\ ** Institute of Information Management (IWI HSG), Univ. of St. Gallen, Switzerland, Email: JanMarco.Leimeister@unisg.ch \\ $\S$ Human-Computer Interaction, Univ. of Stuttgart, Germany, Email: albrecht.schmidt@ vis.uni-stuttgart.de \\ 『 Knowledge and Data Engineering, Univ. of Kassel, Germany, Email: stumme@cs.uni-kassel.de \\ I| Univ. of Kaiserslautern, Germany, Email: zweig@cs.uni-kl.de
}

\begin{abstract}
Today, so-called "smart" or "intelligent" systems heavily rely on machine learning techniques to adjust their behavior by means of sample data (e.g., sensor observations). But, it will be more and more complicated or even impossible to provide those data at design-time of that system. As a consequence, these systems have to learn at run-time. Moreover, these systems will have to self-organize their learning processes. They have to decide which information or knowledge source they use at which time, depending on the quality of the information or knowledge they collect, the availability of these sources, the costs of gathering the information or knowledge, etc. With this article, we propose opportunistic collaborative interactive learning (O-CIL) as a new learning principle for future, even "smarter" systems. O-CIL will enable a "lifelong" or "neverending" learning of such systems in open-ended (i.e., time-variant) environments, based on active behavior and collaboration of such systems. Not only these systems collaborate, also humans collaborate either directly or indirectly by interacting with these systems. The article characterizes $\mathrm{O}$-CIL, summarizes related work, sketches research challenges, and illustrates O-CIL with some preliminary results.
\end{abstract}

\section{INTRODUCTION}

In our future world, technical systems have to evolve over time because they have to function in situations unforeseen at design time. That is, the system has to detect fundamental changes in its environment and react accordingly. This requires that "never-ending" or "lifelong" learning mechanisms have to be implemented into such systems. Amongst other mechanisms (e.g., context- or self-awareness), these learning mechanisms will include appropriate active learning techniques. These future technical systems may be mobile devices, for example, that actively collect data and other kinds of information or knowledge from other devices, humans (who are often nonexperts in a field), or the Internet (e.g., from social networks), cf. Fig. 1 The learning processes comprise large (e.g., thousands), open (participants may leave or others may enter), and heterogeneous (e.g., different types of devices, kinds of knowledge, etc.) groups of "participants" (i.e., smart systems).

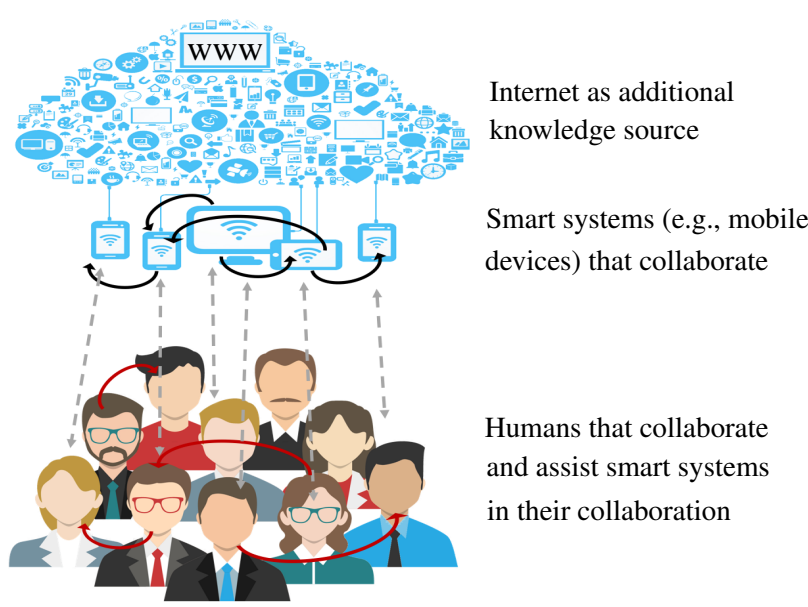

Fig. 1: Idea of opportunistic collaborative interactive learning (O-CIL), from [1].

The data that are labeled may include video, audio, text, or image data. New kinds of human-computer interaction will come into play. Each smart system has to make the best of the available information or knowledge, i.e., it has to learn in an "opportunistic" way. The active gathering of information or knowledge can be seen as a first stage of cooperation of two or more systems, that improve their knowledge to better master their respective tasks. But, these smart systems also offer new possibilities for true collaboration of smart systems and/or humans. As an example, we may consider collaboration processes of humans that are actively supported by collectives of smart systems being aware of the humans' needs and knowledge. As these future smart systems learn "in the wild", we also need new techniques to model and analyze these novel kinds of online learning processes and to guarantee certain properties. 
Developing systems as sketched above requires research in a new field that we call opportunistic collaborative interactive learning (O-CIL). O-CIL can be regarded as an extension of active and collaborative learning for open-ended environments.

In the remainder of this article, we first characterize the concept of O-CIL in more detail (Section III). Then, related work will be summarized in Section IIII In Section IV we introduce the key research challenges of O-CIL and briefly discuss ways to find solutions for the corresponding problems. Preliminary results of a case study that underlines the importance of research in the field of O-CIL are presented in Section V] Finally, Section VI concludes the article.

\section{CHARACTERIZATION OF OPPORTUNISTIC COLLABORATIVE INTERACTIVE LEARNING}

In the early days of computerized technical systems, these systems were specifically designed to master more or less complex tasks. Then, at run-time, they were stuck to these tasks and to the operational environments and situations that were anticipated at design-time. Today, technical systems are able to adapt to new situations, to learn from observations or data they are provided with, and to optimize themselves. For this reason, we call these systems "smart" or "intelligent". In the near future, we will see more and more applications where technical systems that learn to optimize their behavior by means of sample data cannot be provided with all the data they need for learning (or training) at design-time. The reasons for this are manifold, e.g.,

- the sheer amount of data that are necessary to learn mastering a task is too large,

- the temporal effort needed for offline learning would be too large,

- the financial costs to provide the data would simply be too high, or

- the system will encounter new kinds of situations at runtime that cannot be foreseen at design-time.

The latter includes new kinds of operational environments where a "simple" re-parametrization would not be sufficient. Instead, more sophisticated re-structuring mechanisms (e.g., choice of collaboration partners in distributed systems) or the self-adaptation of algorithms that rule a system's own behavior are needed, for instance.

Thus, we need a new generation of smart systems with lifelong (i.e., never-ending) learning capabilities to work in uncertain, open-ended (e.g., time-variant) environments. But, we have to go far beyond the current fields of online learning [2], autonomous learning [3], or organic computing [4]. The systems we need have to act highly autonomous in the sense that

- they assess their own knowledge to decide when this knowledge is not sufficient to cope with new kinds of situations arising at run-time,

- they connect to new information or knowledge sources (e.g., other smart systems) and know which kind of information or knowledge they can obtain from which source,

- they assess the quality of information or knowledge sources and the quality, usefulness, topicality, etc. of information and knowledge they gather,
- they exploit various learning mechanisms to increase their own knowledge, e.g., unsupervised learning, semisupervised learning, transfer learning, reinforcement learning, and, in particular, active learning.

Altogether, this new generation of smart systems will exhibit various self-* properties (e.g., self- and context-awareness or self-reflection [5]) and active learning [6] will play a key role to build such systems. However, the active learning techniques needed here are far beyond the currently available techniques with their limitations regarding number and availability of information sources (typically one that is omnipresent), quality of information sources (typically omniscient), cost for queries (mostly not considered), type of queries (typically only one: labels for data objects), assessment of own knowledge (mostly not regarded), etc. [1].

Above, the term "information source" was used to summarize very different ways to gather the information or knowledge needed for learning. Possible information sources that have to be considered include

- other, similar smart systems that might have similar or complementary tasks (e.g., wearables such as smart watches or smartphones),

- the Internet with its various data bases (e.g., collections of labeled images),

- simulation systems that may be exploited to provide labeled data,

- the own sensors of a system that deliver measurements that are analyzed to reason about the own behavior (e.g., in the case of reinforcement learning), and

- humans that could be asked different kinds of questions (e.g., to label observations, to provide conclusions for rule premises, or to confirm knowledge).

In addition, these systems will keep temporarily unneeded but potentially useful information or knowledge in mind (i.e., their memory) which can be re-used in various ways, e.g., by semi-supervised or transfer learning processes. In different situations, different information and knowledge sources will be accessible or available. Moreover, information and knowledge comes at a cost. While access to the Internet is quite cheap (provided that Internet access is possible), asking humans can be rather costly and should be restricted to rare, but important situations. Altogether, our new generation of smart systems have to combine various sources as well as various learning mechanisms and decide upon an appropriate choice of sources and learning mechanisms depending on current availability, costs, quality, type of query, etc.

Now, we also have to characterize terms such as "information" and "knowledge" for our field of O-CIL more precisely. Here, we adopt the meaning of these terms from the field of data mining [7], [8]. Data mining can be seen as a multi-step process as shown in the data mining pyramid (see Fig. 2, [9]). The idea of this pyramid can briefly be summarized as follows: Raw data are pre-processed to condense application-specific information in attributes or features. Then, knowledge is extracted, e.g., by building classification or regression models based on rules. By analyzing this knowledge off-line it is possible to come to a deeper understanding of its working principles, e.g., by (depending on the knowledge model) analyzing how far premises of contradictory rules 
"overlap" or by determining the fraction of the input space which is "covered" by a certain rule. In a given application, it is possible to gain experience in using the knowledge, e.g., by determining how often a rule is applied or by stating how often it is applied successfully. Understanding and experience will both support the efficient and effective application of the knowledge. Our new generation of smart systems will address these levels as follows, for example:

- The system itself may collect sensor observations and extract relevant attribute values (data and information).

- The operational behavior of the system may be based on classifiers containing rule systems (knowledge).

- Humans can be asked to provide labels for attribute vectors or conclusions for autonomously generated rule premises (information and knowledge).

- Assessing own knowledge before and in action may lead to self-awareness of smart systems regarding the usefulness of knowledge and knowledge deficiencies (understanding and experience).

As understanding and experience can be seen as a kind of meta-knowledge, we will again use the term knowledge in the following when we refer to the levels above data and information.

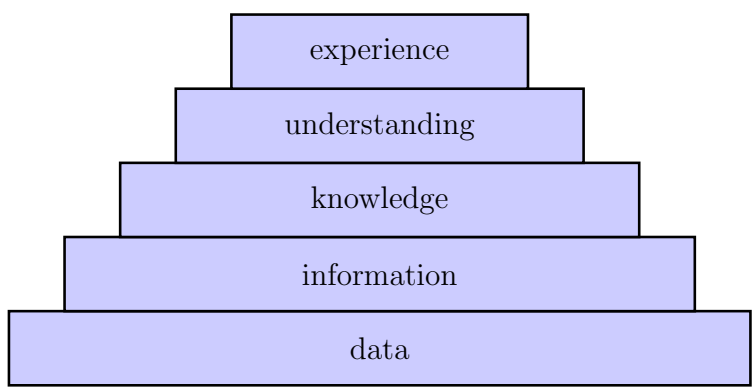

Fig. 2: The data mining pyramid (adopted from [9]).

Our smart systems always have to consider that data, information, and knowledge are uncertain. Here, the meaning of the term uncertainty is adopted from [10]. There, "uncertain" is used as a generic term to address aspects such as "unlikely", "doubtful", "implausible", "unreliable", "imprecise", "inconsistent", or "vague".

The active gathering of information and knowledge can be seen as a first stage of cooperation of two or more systems that improve their knowledge to better master their respective tasks. This effect has a direct impact on the usefulness of such systems for humans. That is, humans profit from smarter devices. However, these smart systems offer new chances to develop truly collaborating smart systems and/or humans:

- Humans may profit from the "collective intelligence" of others (including any combination of other humans, systems, the Internet, etc.).

- Collaboration processes of humans may be actively supported by systems being aware of their respective needs and knowledge.

- Collective systems may solve complex problems that they cannot solve on their own.

These possibilities, however, require appropriate humanmachine interfaces in order to exploit humans as an important and very particular source of information and knowledge and to (pro-)actively provide them with all the information and knowledge they might need (cf. [11]).

"Livelong" (referring here to the lifetime of the technical system) learning is required in open-ended environments, i.e., environments where

- the collective of participating entities (systems, humans, etc.) might be very heterogeneous,

- the number of entities might be large and open in the sense that new entities may enter the collective while others leave,

- the environment in which the entities work might be timevariant, i.e., it changes its properties over time, or

- the entities within the collective may have various tasks that might change over time.

To ensure that our new smart systems do "more good than harm", we also have to develop new techniques (cf. [12]) to analyze such systems, to detect emergent behavior, and to control their behavior in a way that guarantees certain properties that have yet to be defined (cf. the three laws of robotics [13]).

Altogether, we envision a new generation of smart systems based on novel techniques that we call opportunistic collaborative interactive learning $(O-C I L)$ techniques. Learning in such O-CIL based smart systems is

- opportunistic in the sense that these smart systems use all kinds of information and knowledge, even if these sources are sporadically available or uncertain (cf. [14]),

- collaborative in the sense that various humans (experts or non-experts, depending on the application) and/or smart systems collaborate to solve certain problems (e.g., problems that they cannot solve by their own), and

- interactive in the sense that there is an information and knowledge flow not only from humans to the smart systems but also vice versa in various, more or less complex ways.

Closely related to $\mathrm{O}-\mathrm{CIL}$ is dedicated collaborative interactive learning $(D-C I L)$, where the learning process is clearly defined (such as, e.g., in an industrial quality monitoring process), the group of human experts is rather small and they collaborate over a longer period of time (see [1] for details). Research in the field of D-CIL can be seen as a substantial basis for research in O-CIL, but additional challenges need to be overcome (cf. [1]).

Having now abstractly characterized systems with O-CIL capabilities, we now want to briefly sketch some envisioned application scenarios. Of course, not every example will include all the features mentioned above.

1) In the field of cyber-physical systems, connected machine tools learn to cope with new situations arising from sudden events (e.g., tool fracture) or gradual effects (e.g., wear of bearings) by learning from each other, asking an operator, or accessing knowledge in data bases.

2) In the area of ubiquitous computing, wearables such as smart watches or smartphones learn to identify the individual activity patterns of users by asking other mobile 
devices for similar patterns that are already labeled or by sporadically querying a human user.

3) In the field of autonomous robots, robots explore their environment by dynamically combining reinforcement learning techniques with active learning techniques where Internet data bases are asked for labeled images that are similar to the currently observed environment.

4) In the field of autonomous driving, vehicles will gather the information they need from other vehicles, infrastructure (e.g., cameras at crossroads), or the Internet (e.g., highly topical map information). They will cooperate with other vehicles for maneuver and trajectory planning while continuously learning and improving their own behavior.

5) Information security is another area where O-CIL mechanisms will come into play. Distributed intrusion detection systems will encounter threats that they cope with by collaboration. They will actively collect information about new threats and the current situation, also in cooperation with humans.

6) In the field of collaboration engineering, technical systems learn to compose high performing groups of human experts with similar or complementary knowledge. For solving a highly complex task, machines allocate the task to an appropriate group of human experts and support them in their collaborative activities.

The many challenges of O-CIL in smart systems seem to sum up to an overall intractable problem. However, we will address these challenges step by step and sketch specific challenges in Section IV

A representative O-CIL application example, where some preliminary results are already available, will be outlined in some more detail in Section $\mathrm{V}$

\section{RELATED WORK}

The DFG priority program Autonomous Learning [15] can be seen as related to the field of O-CIL, as this program aims to enhance the autonomy of modern learning systems in the sense that the systems independently collect data, choose their learning parameters and representations, and interact with their environment by themselves. For this purpose, autonomous learning combines machine learning techniques from fields such as reinforcement learning, unsupervised learning, semisupervised learning, active learning, and deep learning, for instance. However, the projects of the priority program that use active learning techniques are geared to a very specific application area such as robotics: For example, in [16], an active learning approach is presented that allows a robot to learn dependencies, e.g., the robot can only open a drawer if the key unlocked it before. The approach presented in [17] aims to reduce human-robot interaction by using active learning to teach a robot symbols which abstract from geometric properties of real objects. Another approach described in [18] applies active learning based on streams of 3D point cloud data to train a robot classifying objects. With D-CIL and particular with O-CIL we want to take the next step towards autonomous systems that learn in a collaborative, massively distributed and lifelong manner. For this, we abolish the unrealistic restrictions of active learning and regard learning as a massively distributed (multiple devices, different information and knowledge sources of different and variable quality, etc.) and never-ending task.
The DFG priority program Organic Computing [19] and similar research areas such as Autonomic Computing [20] claimed that the design of open and complex systems requires a paradigm shift in engineering. Each entity of the system needs to be flexible and empowered by major degrees of freedom, because a designer is not able to anticipate all possible configurations at design-time. Only then, the (overall) system is enabled to adapt itself to changing environment conditions at runtime. Organic computing investigates means to develop natureinspired so-called self- $*$ techniques (e.g., self-adaptation, selfconfiguration, self-organization, self-optimization, etc.) for future smart systems. Thus, machine learning plays an important role. Research addressed, for instance, the problem of selfadaptation of systems in noisy, dynamic environments based on Learning Classifier Systems [21]. Evolutionary techniques were used for optimization of a time-variant fitness function. The problem of self-optimization in dynamic and noisy environments (e.g., due to disturbances and anomalies) was also addressed in [22]. There, various challenges (stability-plasticity trade-off, exploration-exploitation problem, stability and safety constraints, etc). were tackled in a holistic way using neurofuzzy techniques. To increase the degree of autonomy in distributed systems novelty and obsoleteness detection techniques for probabilistic classifiers and techniques for collaborative learning in distributed systems based on an exchange of rules contained in such classifiers were proposed [23], [24]. Another approach to increase the autonomy of smart systems is imitation learning. An approach presented in [25] aims at increasing the learning speed in multi-robot societies. Though organic computing is a valuable basis for O-CIL, there are many fundamental research challenges left.

The EU Future and Emerging Technologies (FET) proactive initiative Fundamentals of Collective Adaptive Systems (FoCAS) addresses operating, design, and evolution principles for systems that combine many heterogeneous entities each with its own objectives and policies into ensembles dynamically cooperating over different temporal and spatial scales. A strong focus of the research is on understanding the consequences of mixing humans and computers within flexibly evolving ensembles that replace centralized control with distributed consensus and emphasizes incremental, collaborative diffusion of knowledge over classical learning and data gathering techniques. Key questions are conflict resolution, long term stability, dealing with noisy and outdated information, and handling open ended system configurations. In particular, concepts related to incremental diffusion of knowledge in an environment that lacks central control are highly relevant to the vision of O-CIL. In fact, some of the initial ideas and results build on work done by the authors within FoCAS projects [26], [27]. However, within the FoCAS initiative little attention has been given to fundamental questions of learning, in particular active learning, which are at the core of the novel O-CIL concept.

\section{Research Challenges}

In this section, we will discuss the key research challenges of O-CIL. Firstly, we address the field of active learning, where, amongst others, an overview of appropriate knowledge models and strategies to deal with data of various quality is given (Section IV-A). The challenges arising from dealing with systems that exchange information with concerns related to 
large scale interactions are addressed in the field of collective adaptive systems (Section IV-B]. Thereafter, we focus on the research fields of collaboration engineering and crowdsourcing which provide appropriate mechanisms for designing processes that support interactions between humans and/or smart systems (Section IV-C). In an O-CIL setting, the systems are allowed to ask humans for the importance of attributes of a given application problem. Thus, some research questions related to knowledge acquisition are presented in Section IV-D In order to provide information and knowledge humans have to interact with the learning system, which leads to research issues in the field of human-computer interfaces (Section IV-E). Finally, the field of complex system analysis addresses the problem of analyzing emergent effects in collaboratively learning systems and to control the behavior of the overall system to guarantee certain properties (Section IV-F).

\section{A. Challenges in the Field of Active Learning}

$\mathrm{D}-\mathrm{CIL}$ and in particular O-CIL require new kinds of active learning $(A L)$ techniques. AL mechanisms include, e.g., in the case of pool-based active learning (PAL) [1]:

- a knowledge model that is actively built to solve a regression or classification problem, for instance,

- a learning algorithm, e.g., sequential minimal optimization for a support vector machine (SVM),

- pools of unlabeled and labeled sample data, with decreasing and increasing size, respectively, and

- a selection strategy that decides for which unlabeled samples an oracle (e.g., a human expert) is asked for a label.

Successful AL techniques (e.g., for classification problems) combine generative and discriminative knowledge modeling approaches [28]. Generative knowledge models (e.g., probabilistic models) allow for mechanisms that state knowledge deficits or check for novel or obsolete knowledge, for instance. Discriminative knowledge models such as models based on statistical learning theory (e.g., SVM) focus on solving the classification problem at hand with high accuracy. Selection strategies use information about structure in data taken from generative models to efficiently and effectively solve the classification problem with discriminative models: In an early phase of the AL process, all regions of the input space of the classifier covered with sample data are assigned to classes, while a fine-tuning of the decision boundary is done in a late phase. Regarding the information about structure in data (which can be obtained from unlabeled data) this can be seen as a typical exploration vs. exploitation problem. For an overview of the state-of-the-art in AL see [6], [29], [30], [31], [32], for instance.

Research on O-CIL has to go far beyond the current AL approaches with their limitations (see Section II). Research on D-CIL will be a valuable first step (for research challenges in the field of D-CIL see [1]). At first, we need new kinds of knowledge models and techniques to build these knowledge models as we have different kinds of information and knowledge sources (oracles) with different quality (e.g., expertise). We have to explore the knowledge of different sources and to exploit their knowledge at the same time. We also have to consider the fact that in technical applications, data typically are uncertain. At second, we need new selection strategies as these strategies have to choose not only samples for queries, but also select an appropriate (and available) information and knowledge source and consider the costs coming with that source. Also, other kinds of queries must become possible, e.g., to provide conclusions for rule premises or to confirm or discard knowledge. Moreover, these knowledge modeling techniques and selection strategies have to cope with timevariant environments and possibly changing tasks in openended environments which requires new combinations of PAL and stream-based AL (SAL) techniques. We can say that $\mathrm{O}-$ CIL has roots in organic computing, too, as the new generation of smart collaborating systems have to self-organize their own (active) learning process.

The field of ensemble learning [33], [34], where several learning algorithms and/or models are combined to increase the performance, e.g., of a classifier or a regression model, is also related to $\mathrm{O}-\mathrm{CIL}$.

\section{B. Challenges in the Field of Collective Adaptive Systems}

As already pointed out, O-CIL is heavily influenced by organic computing. Thus, it combines the general active learning related challenges with concerns related to large scale interaction effects and emergent behavior. In previous work we have already shown that systems, in which learning takes place incrementally through information exchange based on "meeting" patterns between smart systems, phase transition effects can be observed [26]. The general idea is that two systems, who have exchanged knowledge to improve the quality of their local classifiers, are able to improve the knowledge of other systems they interact with even further, effectively creating an overall feedback-loop-driven non-linear dynamic system. In general, such feedback loops can be positive, ineffectual, or negative (if disinformation is spread) and need to be carefully controlled. Of particular interest are systems where the information exchange patterns depend on human behavior which is in turn dependent on the information that the system provides. This is, for example, the case in collaborative indoor location systems [35].

\section{Challenges in the Fields of Collaboration Engineering and Crowdsourcing}

D-CIL and O-CIL require an interaction between humans and smart systems. The research fields of collaboration engineering and crowdsourcing provide appropriate mechanisms for systematically designing processes supporting such interactions. Collaboration engineering is an approach for designing collaborative processes for solving highly complex recurring tasks and conducting them without the ongoing help of a human moderator. The process design leads human experts through several structured activities and supports them in their collaboration with each other for solving a task [36], [37], [38]. With respect to an unknown number of humans and tasks, crowdsourcing research provides additional insights. In that context, a task is outsourced to an undefined, generally large group of people in the form of an open call [39]. In general, crowdsourcing is characterized by three roles: crowdsourcer (e.g., human expert who defines a task), crowdsourcingplatform (e.g., system which offers human workers the tasks), crowdsourcees (e.g., human workers who solve the task and 
receive compensation) [39]. To bring the roles together, crowdsourcing comprises five consecutive phases (cf. [39]):

- Phase 1 - Task specification: Definition of task granularity and a clear task description with expectations regarding the solution.

- Phase 2 - Selection of appropriate crowdsourcees: Decision for one of two selection mechanisms, either an open call (all crowdsourcees have the chance to participate) or a selection of appropriate crowdsourcees (analyzing the skills of potential crowdsourcees).

- Phase 3 - Execution of the task: Decision for one of three mechanisms for solving the task: crowdvoting (crowdsourcee makes a vote), crowdfunding (crowdsourcee makes an investment), or crowdcreation (crowdsourcee develops a complex concept).

- Phase 4-Aggregation and selection of solutions: Decision for one of two selection mechanisms: consolidating the several solutions (integrative) or selecting the most appropriate solution (selective).

- Phase 5 - Compensation: Depending on the decision of phase 4, all crowdsourcees who submitted a solution (integrative) or only the crowdsourcee whose solution was selected (selective) receive compensation.

Transferring the ideas and principles from collaboration engineering and crowdsourcing to D-CIL and O-CIL provides several new questions. First, we need to identify situations, in which smart systems take over the role of a human crowdsourcer. It is important to analyze the types of tasks which exist in the context of D-CIL and O-CIL. This leads to questions addressing requirements for defining and structuring a task in order to allow further automated processing by smart systems. Furthermore, it is important to develop appropriate frameworks for describing situations in which smart systems become able to define and to structure a task for further processing. Second, we need to identify the challenges for enhancing a smartsystem-driven collaboration between the crowdsourcer and the crowdsourcees as well as among the crowdsourcees. In that context, it is necessary to identify the appropriate granularity of a task and its expected solution. Based on these insights, we need to develop mechanisms which help smart systems to distribute the task to the crowdsourcees. In addition, we need new mechanisms which support smart systems in allocating the task to crowdsourcees (e.g., human experts or intelligent systems), who have the required knowledge to solve the task. This also comes along with questions focusing on mechanisms for composing a group of humans who should solve the task. In that context, collaborative work practices among crowdsourcees (e.g., a group of human experts, intelligent systems, or hybrid forms) need to be designed in order to ensure an optimal solution.

\section{Challenges in the Field of Knowledge Acquisition}

Within the AI community, there are different perspectives on the area of (collaborative) interactive learning. While the understanding from a machine learning perspective is to allow machine learning algorithms to ask a human once in a while, whenever they require some specific additional training data, knowledge acquisition uses the opportunity to alleviate a human in the-predominantly manual-knowledge acquisition task whenever possible by automatizing answers as much as possible. A specific knowledge acquisition technique is attribute exploration [40]. Its combination with machine learning has been studied successfully, because of its formal mathematical foundation.

For a given domain of interest, attribute exploration is generating implications for a given set of attributes. For each generated implication, the algorithm is asking the expert if the implication holds for all objects of the domain of interest or not. The expert either accepts the implication or has to provide a counter-example. For instance, if we are interested in the countries of the world, the expert might be asked whether "OECD member" $\rightarrow$ "safe state of origin" holds or not. She/He has either to accept the implication or she/he has to provide a counter-example. The algorithm guarantees that the number of accepted implications is always minimal. Some of the questions may be answered automatically by relying on existing background knowledge [41], [42], or by revoking a reasoning mechanism [43]. The task of identifying counterexamples can be alleviated by extending attribute exploration with web search [44].

Answering the questions of the algorithm is a tedious task. We are therefore looking for solutions that distribute this task to several persons. In a dedicated setting (D-CIL), one could eventually modify the algorithm such that it generates an alert when answers of different members of an expert group become inconsistent. In that case, one might expect that the experts negotiate (eventually supported by the system) a common understanding of the problem. In an opportunistic setting (OCIL) - e.g., when the answers are crowdsourced-one has to encounter answers of varying quality. This brings up the following research questions (cf. also Section IV-A):

1) Which expert should be asked? Who is most likely to provide an answer at all, a correct answer, and/or the best counter-example?

2) Should one ask more than one expert for each question?

3) How to deal with inconsistent answers? Should they all be stored and made explicit when presented to the user? How do they influence the knowledge acquisition procedure? Or can the inconsistency be resolved in a negotiation process of the experts, supported by the exploration procedure?

4) How can the procedure handle the correction of a previously given answer?

5) Can one extend the procedure such that the learned model is close enough to the true (unknown) model within a certain probability?

\section{E. Challenges in the Field of Human-Computer Interfaces}

The degree to which humans are able and willing to provide meaningful input is central to the performance of an O-CIL system. Largely, both the ability and the willingness depends on an appropriate interaction paradigm. The three main issues to be considered are (1) interruptibility, (2) an appropriate input modality adapted to the context situation, and (3) a model of the learning process allowing the user to easily understand the impact of the information she/he provides.

Detecting when and how it is appropriate to interrupt the users asking for input is a well known open problem in mobile and wearable computing [45], [46]. In general, there are few 
situations in which asking the user for input is categorically out of question. Instead, each request is associated with a cost, which in turn relates to the level of response that the user is likely to provide. Models of such cost involve user preferences, her/his activity, the specific situation, the social setting, the importance of the cause (why the input is asked) to the user, and the effort involved in answering the request. Various approaches have been investigated towards building such cost models. Much early work [47] focused on statistical learning, which however, has the disadvantage of requiring labeled data. Other approaches combine appropriate user models with context recognition or use response rate/quality as "labels". Within O-CIL systems, the evolution of such cost models will also be done in a collaborative manner, raising questions of transferability of users' models, embedding the cost model learning into social interactions, and the relationship between the learning of the user models and the main learning task.

Clearly, the cost of requests can be reduced if they can be achieved in a minimally disruptive way. A major problem of labeling data for real world activity recognition stems from the complexity of the required input. In general, it is not binary but may include the introduction of new classes or explanation of complex activity or context hierarchies. How to map such onto simple modalities such as, for example, the micro interactions of Google Glass [48] is an open question. Again, for O-CIL the question of collaboration in different settings needs to be taken into account.

A final challenge is how to communicate the importance and effect of different types of input to the user (cf. also Sections IV-A and IV-D). Optimally, the user should be given an understanding of how her/his cooperation in different situations impacts the future performance of the system (which in turn impacts the quality of service that the system will be providing to her/him in the future). This does not necessarily imply that a user must be given an in-depth understanding of the actually workings of the system. Instead a simple, intuitive mental model is required. Such models are also important to go beyond the mere acquisition of individual labels towards more elaborate involvement of users in the improvement of a classifier (e.g., suggesting where to collect data next, which sensors to use, etc).

\section{F. Challenges in the Field of Complex Systems Analysis}

Collaboration between humans is based on a relationship such as friendship or trust in each others' work, which can be represented and analyzed as a complex network [49]. A collaboration network can either be initiated by a common project, such as Wikipedia or projects on github, or an existing network can decide to collaborate to produce something. In both cases, very distinct network structures emerge that influence the efficiency of communication and productivity. A central question is now how smart devices can engage humans in a collaboration with a beneficial network structure or-in case that the collaboration is based on an already existing network structure-how to support the communication on this underlying network such that it is most efficient. One example for an inefficient but common network structure is the so-called small-world network structure that consists of a mainly local grid structure plus some long-range connections [50]. Here, collaboration in a repeated Prisoner's dilemma is less likely to be achieved [51]. To achieve an efficient communication on such a network structure, long-range edges need to be identified by the system and communication over them discouraged. Similarly, another common network structure contains a scale-free degree distribution; in comparison to a Poissonian distributed degree distribution, it decreases the robustness in case of attacks against high-degree nodes and enhances its robustness against random attacks [52]. Supporting new connections within the collaboration network that drive the network towards a Poissonian or scale-free degree distribution can thus influence the robustness of the network under different types of failure, noise, or attacks [53]. But here the question is how the smart systems can learn which persons would actually connect with each other since humans build their networks not only based on competencies but also on psychological properties which need to be regarded [54]. In summary, existing network structures between collaborating persons have to be known and analyzed in order to support an efficient network structure for communication and collaboration. This has to be done reliably and in real-time in a dynamic and noisy sensoric environment while the systems learn psychological, time- and environment dependent parameters that may be deeply human such as the propensity that two persons will work with each other dependent on their gender, race, culture, and education, to name just a few of the possibly determining parameters.

\section{CASE Study}

Some of the concepts underlying D-CIL or O-CIL have already been explored in practice, in a cooperative travel time estimation application. Based on an urban mobility simulation built on data extracted from the real world city of Trento, Italy, we explored both strategies for modeling and retaining a distributed store of knowledge as well as resulting system behaviors when different learning and exchange strategies were applied. In this section, we will first give a short description of the simulator used. Then, we will present an outline of the knowledge and exchange model. Finally, we will show some preliminary results we obtained.

The simulator we used for this case study was built upon the Netlogo multi-agent simulation toolkit [55]. Netlogo forms the component driving the actual simulation (i.e., progressing time for the agents) and provides a convenient basis for handling user interface based input and output of data. On top of that, we designed a library encapsulating all the actual real world information. This includes data acquired from the municipality of Trento, such as traffic patterns, utilization of parking spaces across the city, bus routes, and various regions of interest (or different behavior) such as industrial or commercial zones. It also includes a wealth of additional data acquired by questionnaires filled out by residents of the Trento area. Finally, the simulation uses some external services, among them a multi-modal journey planner available through the city, capable of providing whole itineraries for reaching a destination specified by a user. By generating an agent set based roughly upon the demographic properties of the larger Trento area and supplying some basic behavioral rules (such as workers going to work in the morning from residential to industrial zones), a reasonably realistic simulation of urban traffic could be constructed. It should be noted that the aim was not to create a model of Trento itself, but rather to provide a real-world inspired test environment for our concepts (for a 
more comprehensive explanation, see [56]). The underlying task model that describes what each agent is actually doing to achieve its goals is also very flexible. From a modeling perspective, the starting point is an abstract goal that is consecutively refined down to concrete atomic actions, which are then executed in sequence. An example: An agent may start with a goal of going from A to B. This may be refined to: "walk from A to A1", "take the bus from A1 to B1", "walk to B". The middle leg is then refined again to "stay on bus from A1 to A2, A2 to A3, A3 to B1". We believe that this technique is a reasonable starting point for the challenges presented in Section IV-C

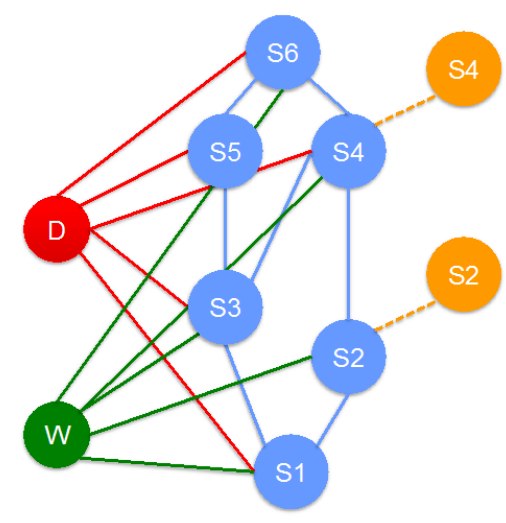

Fig. 3: Example of a graph based knowledge model for the case study.

The knowledge model, which each of the 40.000 agents representing one person or smartphone applied, was based on a graph representation. As can be seen in Fig. 3, there were different types of nodes: $\mathrm{D}$ and $\mathrm{W}$ represent context factors (time of day and weather, respectively), while S1 to S6 represent nodes derived directly from the street graph underlying the simulation. S2 and S4 connected by dotted lines represent temporal aspects, e.g., the load on a street segment one temporal unit (e.g., 15 minutes) earlier. Edges represent a significant dependence of a node on the state of a connected other one. In general, for the urban mobility scenario, the model consisted of three kinds of vertices: context (usually connected to most other nodes), nodes derived from the underlying traffic simulation (street segments, bus stops), with connections mostly implied by spatial proximity, and temporal nodes modeling the influence of previous states. Learning was accomplished by adding information about the state of visited nodes collected during actual journeys performed within the system. There are similarities here to conditional random fields and, via that, to Hidden Markov Models and Bayesian Networks. Thus, this kind of model can be tuned towards both generative as well as discriminative refinements or even a combination of both. As mentioned in Section IV-A this is an important consideration for O-CIL.

Knowledge exchange was based upon a shared underlying knowledge about the structure of the model itself. Even though each individual agent might not have any knowledge about a specific node, the node itself can readily be identified (e.g., as context node "weather" or street segment S1). Thus, when agents exchange or fuse knowledge (e.g., based on physical proximity or some other notion of closeness), many different cases can be considered, for example:

- nodes known by both and connected by edges in both models (here, the new nodes and/or edges are given by a weighted fusion) or

- nodes and edges only known by one agent (here, the information can be incorporated into the less knowledgeable model).

It should be noted that the exchange paradigms used in the model are still very preliminary. They do, however, already hint at the interesting aspects presented in Section IV-F The actual knowledge exchange can be tuned to conform to various requirements. In the simplest case, agents share their entire graph. In more complex scenarios, this may be reduced to a subset (e.g., for privacy or efficiency reasons) or even just the answers to specific queries (e.g., agent A asking agent B "Can you tell me how to get from A to B via bus?"). The advantages and disadvantages of each possibility are also inherently linked to the challenges presented in Section IV-D When agents are presented with multiple choices (e.g., "take the car", "walk", "take the bus"), decisions are made based on an utility metric that takes into account different factors such as travel time and cost. While the system itself-without any accumulated knowledge - can give rough estimates, it specifically cannot take into consideration events such as morning or evening rush hours. Thus, as one performance metric, we looked at how the quality of the travel time estimate changes based on different scenarios: In three runs of six simulated days, agents either did not learn at all, only learned locally, or shared their knowledge globally. As can be seen in Fig. 4, the system without any knowledge retention actually underestimated travel time every day (since it could not incorporate any historic knowledge). The local learning converged towards a reasonable estimate, while the global knowledge repository actually showed a slight fluctuation around a mean error of zero.

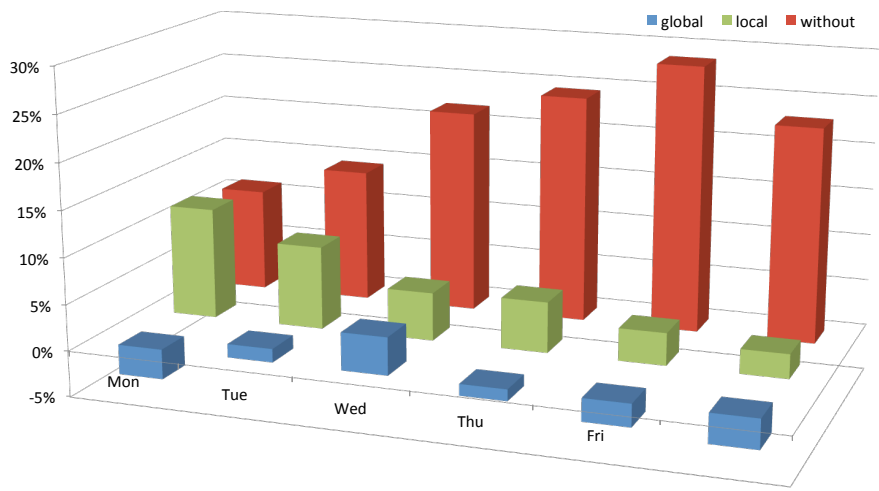

Fig. 4: Travel time estimate error for different learning paradigms (six days, 3 paradigms).

In a second series of experiments, where we simulated two days, a fourth scenario was added: Agents again shared their knowledge, but only on a regional (instead of a global) basis. Fig. 5 shows that the regional model tends to behave similar to the global one, but is a bit more prone to fluctuation. Referring back to the challenges stated in Section IV-B, this 
basic simulation setup can already be used to highlight a few interesting questions. For example, given enough penalty for tardiness, will there be a phase transition to a transport ecosystem dominated by public options? Or maybe a fluctuating system where traffic jams cause people to switch to buses, but overfilled buses drive people back to (at that time) less congested roads? Also, this scenario is ideally suited to study information propagation, especially when considering misinformation (that can easily be purposefully injected).

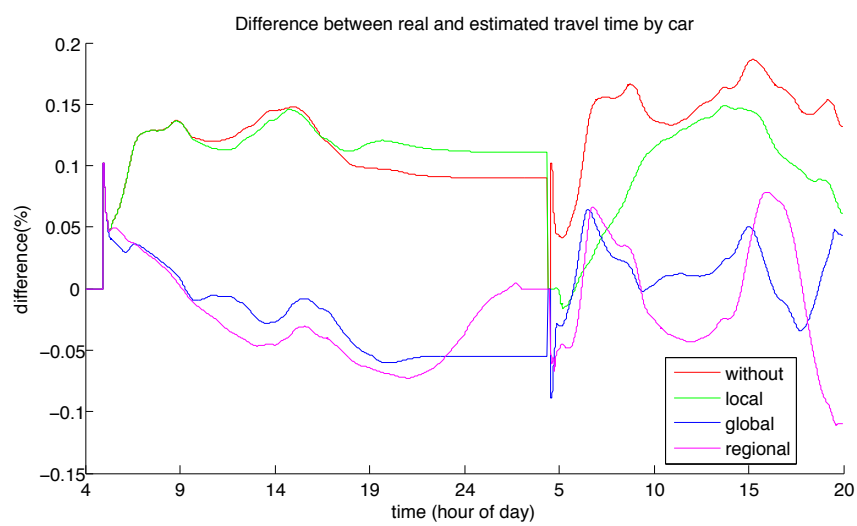

Fig. 5: Travel time estimate error for different learning paradigms (two days, 4 paradigms).

Overall, even though these results are preliminary, they are already interesting: In the interaction case (where agents actually share knowledge with each other), there is an oscillating effect not seen in the strictly local learning. Also, the effect seems to be higher with regional interaction vs. global knowledge exchange. This provides a solid foundation for a lot of future research possibilities: More comprehensive simulations are necessary, better strategies have to be developed (especially opportunistic ones), new performance indices must be defined, the approach has to be formalized, etc. However, these preliminary results already show that O-CIL will provide important contributions for future smart systems engineering.

\section{SUMMARY}

A key limitation of systems that aim to learn to make sense out of real-life unconstrained situations is the need for extremely large amounts of training data. This amount of data reflects the diversity and complexity of the real world. Our long term vision for solving this problem is motivated by the observation that increasingly nearly every human being on the planet is equipped with devices that can continuously collect sensor data. Even if each person provides only very limited number of labels, over time enough labeled data to cover high degree of complexity and diversity can be collected. However, for many reasons (privacy, resources, communication) just globally collecting data from every human being on the planet for a centralized global training data repository is neither feasible nor desirable. Instead, we propose O-CIL, a novel learning paradigm that leverages social interactions and structures to incrementally accumulate knowledge. We have shown in this article how such an approach requires a combination of methods from, amongst others, machine learning, knowledge discovery, human computer interaction, collective adaptive systems, collaboration engineering, and complexity science.

\section{REFERENCES}

[1] A. Calma, J. M. Leimeister, P. Lukowicz, S. Oeste-Reiß, T. Reitmaier, A. Schmidt, B. Sick, G. Stumme, and A. K. Zweig, "From active learning to dedicated collaborative interactive learning," in ARCS 2016, 29th International Conference on Architecture of Computing Systems, Workshop Proceedings, A. L. Varbanescu, Ed. Nuremberg, Germany: VDI Verlag, Berlin, 2016, pp. 1-8.

[2] O. Fontenla-Romero, B. Guijarro-Berdiñas, D. Martinez-Rego, B. Pérez-Sánchez, and D. Peteiro-Barral, "Online machine learning," in Efficiency and Scalability Methods for Computational Intellect, B. Igelnik and J. M. Zurada, Eds. Hershey, PA: IGI Publishing, 2013, pp. 27-54.

[3] "Future challenges for the science and engineering of learning." Technical report, National Science Foundation workshop, 2007, (last access: 04/07/2016). [Online]. Available: http://cnl.salk.edu/Media/ NSFWorkshopReport.v4.pdf

[4] C. Müller-Schloer, H. Schmeck, and T. Ungerer, Eds., Organic Computing - A Paradigm Shift for Complex Systems. Basel, Switzerland: Birkhäuser Verlag, 2011.

[5] S. Tomforde, J. Hähner, S. von Mammen, C. Gruhl, B. Sick, and K. Geihs, "Know Thyself - Computational Self-Reflection in Intelligent Technical Systems," in 8th IEEE Int. Conf. on Self-Adaptive and SelfOrganizing Systems Workshops, SASOW 2014, London, UK, 2014, pp. $150-159$

[6] T. Reitmaier and B. Sick, "Let us know your decision: Pool-based active training of a generative classifier with the selection strategy 4DS," Information Sciences, vol. 230, pp. 106-131, 2013.

[7] D. Fisch, E. Kalkowski, and B. Sick, "In Your Interest: Objective Interestingness Measures for a Generative Classifier," in Proceedings of the 3rd International Conference on Agents and Artificial Intelligence (ICAART 2011), Rome, Italy, 2011, pp. 414-423.

[8] U. Fayyad, G. Piatetsky-Shapiro, and P. Smyth, "The kdd process for extracting useful knowledge from volumes of data," Communications of the ACM, vol. 39, no. 11, pp. 27-34, 1996.

[9] M. J. Embrechts, B. Szymanski, and K. Sternickel, "Introduction to scientific data mining: Direct kernel methods and applications," in Computationally Intelligent Hybrid Systems, S. J. Ovaska, Ed. Piscataway, NJ: IEEE Press, 2005, ch. 10, pp. 317-362.

[10] A. Motro and P. Smets, Eds., Uncertainty Management in Information Systems - From Needs to Solutions. Springer US, 1997.

[11] A. Schmidt, "Following or leading?: the HCI community and new interaction technologies," interactions, no. 22, pp. 74-77, 2015.

[12] M. Kaufmann and K. Zweig, "Modeling and designing real-world networks," in Algorithmics of Large and Complex Networks, J. Lerner, D. Wagner, and K. Zweig, Eds. Springer Berlin Heidelberg, 2009, vol. 5515, pp. 359-379.

[13] I. Asimov, I, Robot, ser. Robot series. Bantam Books, 1950.

[14] D. Roggen, G. Tröster, P. Lukowicz, A. Ferscha, J. del R. Millan, and R. Chavarriaga, "Opportunistic human activity and context recognition," Computer, vol. 46, no. 2, pp. 36-45, 2013.

[15] M. Toussaint, H. Ritter, J. Jost, C. Igel, S. Behnke, M. Bethge, B. Hammer, M. Riedmiller, R. Rojas, W. von Seelen, and J. Triesch, "DFG Priority Programmme 1527 Autonomous Learning," http: //autonomous-learning.org (last access 28/04/2016).

[16] J. Kulick, S. Otte, and M. Toussaint, "Active exploration of joint dependency structures," in IEEE International Conference on Robotics and Automation (ICRA'15), Seattle, WA, USA, 2015, pp. 2598 - 2604.

[17] J. Kulick, M. Toussaint, T. Lang, and M. Lopes, "Active learning for teaching a robot grounded relational symbols," in Proceedings of the 23rd International Joint Conference on Artificial Intelligence (IJCAI'13), Beijing, China, 2013, pp. $1-7$.

[18] A. Narr, R. Triebel, and D. Cremers, "Stream-based active learning for efficient and adaptive classification of $3 \mathrm{~d}$ objects," in IEEE International Conference on Robotics and Automation (ICRA'16), Stockholm, Sweden, 2016, p. (to appear). 
[19] C. Müller-Schloer, "Organic Computing: On the Feasibility of Controlled Emergence," in CODES+ISSS'04 Proceedings of the 2nd International Conference on Hardware/software codesign and system synthesis. New York, NY: ACM, 2004, pp. 2-5.

[20] J. O. Kephart and D. M. Chess, "The Vision of Autonomic Computing," IEEE Computer, vol. 36, no. 1, pp. 41-50, 2003.

[21] E. Cakar, N. Fredivianus, J. Hähner, J. Branke, C. Müller-Schloer, and H. Schmeck, "Aspects of learning in OC systems," in Organic Computing - A Paradigm Shift for Complex Systems, C. Müller-Schloer, H. Schmeck, and T. Ungerer, Eds. Birkhäuser, 2011, ch. 3.1, pp. 237251.

[22] W. Brockmann, N. Rosemann, and E. Maehle, "A framework for controlled self-optimization in modular system architectures," in Organic Computing - A Paradigm Shift for Complex Systems, C. Müller-Schloer, H. Schmeck, and T. Ungerer, Eds. Birkhäuser, 2011, ch. 3.4, pp. 281294.

[23] D. Fisch, M. Jänicke, E. Kalkowski, and B. Sick, "Techniques for Knowledge Acquisition in Dynamically Changing Environments," ACM Tr. on Autonomous and Adaptive Systems, vol. 7, no. 1, 2011.

[24] - "Learning from others: Exchange of classification rules in intelligent distributed systems," Artificial Intelligence, vol. 187-188, pp. 90-114, 2012.

[25] A. Jungmann, B. Kleinjohann, and W. Richert, "Increasing learning speed by imitation in multi-robot societies," in Organic Computing - A Paradigm Shift for Complex Systems, C. Müller-Schloer, H. Schmeck, and T. Ungerer, Eds. Birkhäuser, 2011, ch. 3.5, pp. 295-307.

[26] G. Kampis and P. Lukowicz, "Collaborative knowledge fusion by adhoc information distribution in crowds," Procedia Computer Science, vol. 51, pp. 542-551, 2015.

[27] G. Kampis, T. Franke, S. Negele, and P. Lukowicz, "Efficient information distribution using human mobility," Procedia Computer Science, vol. 66, pp. 382-391, 2015.

[28] C. M. Bishop, Pattern Recognition and Machine Learning. New York, NY: Springer, 2006.

[29] B. Settles, "Active learning literature survey," University of Wisconsin, Department of Computer Science, Computer Sciences Technical Report 1648, 2009.

[30] T. Reitmaier, A. Calma, and B. Sick, "Transductive active learning a new semi-supervised learning approach based on iteratively refined generative models to capture structure in data," Information Sciences, vol. 293, pp. 275-298, 2015.

[31] J. Jiang and H. H. S. Ip, "Active learning with SVM," in Encyclopedia of Artificial Intelligence, J. Ramón, R. Dopico, J. Dorado, and A. Pazos, Eds. Hershey, PA: IGI Global, 2009, vol. 3, pp. 1-7.

[32] J. Kremer, K. S. Pedersen, and C. Igel, "Active learning with support vector machines," Wiley Interdisciplinary Reviews. Data Mining and Knowledge Discovery, vol. 4, no. 4, pp. 313-326, 2014.

[33] D. Opitz and R. Maclin, "Popular ensemble methods: An empirical study," Journal of Artificial Intelligence Research, vol. 11, pp. 169198, 1999.

[34] G. Brown, J. Wyatt, R. Harris, and X. Yao, "Diversity creation methods: a survey and categorisation," Information Fusion, vol. 6, no. 1, pp. 5-20, 2005.

[35] G. Kampis, J. W. Kantelhardt, K. Kloch, and P. Lukowicz, "Analytical and simulation models for collaborative localization," Journal of Computational Science, vol. 6, pp. 1-10, 2015.

[36] O. R. Briggs, G. L. Kolfschoten, G.-J. de Vreede, and D. L. Dean, "Defining key concepts for collaboration engineering," in Proceedings of the Twelfth Americas Conference on Information Systems, Acapulco, Mexico, 2006, pp. 121-128.

[37] S. Oeste-Reiß, M. Söllner, and J. M. Leimeister, "Development of a peer-creation-process to leverage the power of collaborative knowledge transfer," in 49th Hawai International Conference on System Sciences (HICSS), Koloa, HI, 2016, pp. 797-806.

[38] E. A. C. Bittner and J. M. Leimeister, "Creating shared understanding in heterogeneous work groups: Why it matters and how to achieve it," Journal of Management Information Systems, vol. 31, no. 1, pp. 111$-144,2014$.

[39] J. M. Leimeister, S. Zogaj, and D. Durward, "New forms of employment and IT-crowdsourcing," in 4th Conference of the Regulating for Decent Work Network, Geneva, Switzerland, 2015, pp. 1-10.

[40] B. Ganter and S. Obiedkov, Conceptual Exploration. Berlin Heidelberg: Springer, 2016.

[41] G. Stumme, "Attribute exploration with background implications and exceptions," in Data Analysis and Information Systems. Statistical and Conceptual approaches. Proc. GfKl'95. Studies in Classification, Data Analysis, and Knowledge Organization 7, H.-H. Bock and W. Polasek, Eds. Heidelberg: Springer, 1996, pp. 457-469.

[42] B. Ganter, "Attribute exploration with background knowledge," Theoretical Computer Science, vol. 217, no. 2, pp. 215-233, 1999.

[43] F. Baader, B. Ganter, B.Sertkaya, and U. Sattler, "Description logic knowledge bases using formal concept analysis," in Proceedings of the Twentieth International Joint Conference on Artificial Intelligence (IJCAI'07), M. M. Veloso, Ed., 2007, pp. 230-235.

[44] R. Jäschke and S. Rudolph, "Attribute exploration on the web," in Contributions to the 11th International Conference on Formal Concept Analysis, P. Cellier, F. Distel, and B. Ganter, Eds., Dresden, Germany, 2013, pp. 19-34.

[45] J. Ho and S. S. Intille, "Using context-aware computing to reduce the perceived burden of interruptions from mobile devices," in Proceedings of the SIGCHI conference on Human factors in computing systems. New York, NY: ACM, 2005, pp. 909-918.

[46] A. Schmidt, M. Beigl, and H.-W. Gellersen, "There is more to context than location," Computers \& Graphics, vol. 23, no. 6, pp. 893-901, 1999.

[47] N. Kern and B. Schiele, "Context-aware notification for wearable computing," in Proceedings of the Seventh International Symposium on Wearable Computers (ISWC 03). IEEE, 2003, pp. 223 - 230.

[48] T. Starner, "Project glass: An extension of the self," Pervasive Computing, IEEE, vol. 12, no. 2, pp. 14-16, 2013.

[49] S. Wasserman and K. Faust, Social Network Analysis - Methods and Applications, revised, reprinted ed. Cambridge University Press, Cambridge, 1994.

[50] D. J. Watts and S. H. Strogatz, "Collective dynamics of 'small-world' networks," Nature, vol. 393, pp. 440-442, June 1998.

[51] D. J. Watts, Six Degrees - The Science of a Connected Age. New York, NY: W.W. Norton \& Company, 2003.

[52] R. Albert, H. Jeong, and A.-L. Barabási, "Error and attack tolerance of complex networks," Nature, vol. 406, pp. 378-382, 2000.

[53] K. A. Zweig and K. Zimmermann, "Wanderer between the worlds self-organized network stability in attack and random failure scenarios," in Proceedings of the 2nd SASO. Venice, Italy: IEEE, 2008, pp. 309318.

[54] M. McPherson, L. Smith-Lovin, and J. M. Cook, "Birds of a feather: Homophily in social networks," Annual Review of Sociology, vol. 27, pp. 415-444, 2001.

[55] U. Wilensky, "Netlogo," http://ccl.northwestern.edu/netlogo/ 1999, (last access: 05/11/2016), Center for Connected Learning and ComputerBased Modeling, Northwestern University. Evanston, IL.

[56] A. Poxrucker, G. Bahle, and P. Lukowicz, "Simulating adaptive, personalized, multi-modal mobility in smart cities," in Proceedings of EAI International Conference on Smart Urban Mobility Services. Toronto, ON: Springer, 2015. 